\title{
Comparison of algorithms for identification of IIR sys- tems from binary measurements on the output
}

\author{
Hicham Oualla ${ }^{1,2, *}$, Mathieu Pouliquen ${ }^{1}$, Miloud Frikel $^{1}$, and Said Safi ${ }^{2}$ \\ ${ }^{1}$ Laboratoire d'Automatique de Caen - EA 7478 Normandie Univ, UNICAEN, ENSICAEN, LAC, \\ Caen, France \\ ${ }^{2}$ Department of Mathematics and Informatics, University Sultan Moulay Slimane, Beni Mellal, Mo- \\ rocco
}

\begin{abstract}
In the last decade, a significant number of algorithms of systems using only a quantized output, has appeared. In this study, an overview of identification methods of IIR systems, using quantized or binary output, is presented. A short description of the methods existing in the literature is given. The methods are compared both on the basis of simulation examples.

Keywords: Real-time identification, IIR systems, Binary output.
\end{abstract}

\section{Introduction}

The identification of systems using quantized or binary output has been an important research topic for several years. The use of quantized or binary output introduces difficulties, since only very limited information is avaible for system identification. Despite this limitation different interesting methods have been proposed so far.

The main motivation is found in many applications and system, due mostly to the recent explosive development in computer and communication networks, as ATM network, which only the quantized (or binary) output observations are available. Various of quantized / binary estimation algorithms are developed, most of these solutions are designed for Finite Impulse Response (FIR) systems, as methods proposed in [19], [1], [2], [3], [4], [5], [6], [7] and [8]. Some methods are dedicated to Infinite Impulse Response (IIR) systems as in [9], [10],[11],[12], [14], [13], and [15]. others are dedicated to non-linear system.

In this paper we consider those dedicated to identification of IIR systems ( Output-Error model structure). In order to propose a first comparison of these methods.

The rest of the paper is organized as follows: the identification problem is formulated in Section II. The main algorithms of the literature are described in Section III. These algorithms are tested and compared on numerical simulation, results are given and commented in section IV. Section V concludes the paper.

\footnotetext{
*e-mail: ouallahicham93@gmail.com
} 


\section{Problem statement}

In this section, some notations and assumptions that will be used throughout the paper are introduced. We consider here the discrete-time linear system depicted on Fig. 1. The system is described by:

$$
\left\{\begin{array}{l}
x_{t}=G^{*}(q) u_{t} \\
y_{t}=x_{t}+n_{t} \\
s_{t}=Q_{c}\left(y_{t}\right)
\end{array}\right.
$$

where $u_{t}$ is the input of the linear system. $n_{t}$ is an additive noise.

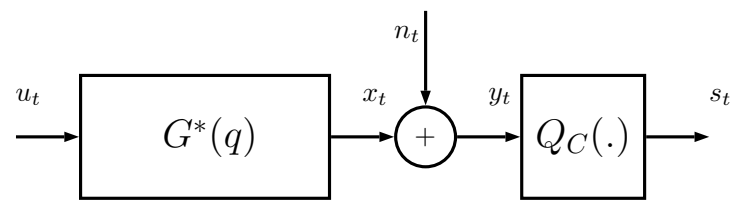

Figure 1. The considered linear system with binary measurements on the output

$G^{*}(q)$ is the linear transfer function described as follows

$$
G^{*}(q)=\frac{B^{*}(q)}{A^{*}(q)}
$$

where

$$
\begin{aligned}
& A^{*}(q)=1+a_{1}^{*} q^{-1}+\cdots+a_{n_{a}}^{*} q^{-n_{a}} \\
& B^{*}(q)=b_{0}^{*}+b_{1}^{*} q^{-1}+\cdots+b_{n_{b}}^{*} q^{-n_{b}}
\end{aligned}
$$

$G^{*}(q)$ is assumed to be stable. $q^{-1}$ is the backward shift operator such that $q^{-1} u_{t}=u_{t-1} . n_{a}$ and $n_{b}$ are the degrees of respectively $A^{*}(q)$ and $B^{*}(q)$.

$y_{t}$ is measured with the binary sensor $Q_{C}($.$) as follows$

$$
s_{t}=Q_{C}\left(y_{t}\right)=\left\{\begin{array}{l}
1, \text { if } y_{t} \geq C \\
-1, \text { otherwise }
\end{array}\right.
$$

where $C$ is a given threshold.

Let define the following vector $\theta^{*} \in R^{n}$ with $n=n_{a}+n_{b}+1$ :

$$
\theta^{*}=\left(\begin{array}{c}
a_{1}^{*} \\
\cdots \\
a_{n_{a}}^{*} \\
b_{0}^{*} \\
b_{1}^{*} \\
\cdots \\
b_{n_{b}}^{*}
\end{array}\right)
$$

Objective: The objective of the paper is to propose a comparison of algorithms proposed in literature for the identification of $G^{*}(q)$ given $\left\{s_{t}, u_{t}\right\}_{t=1}^{N}$ generated by (1) and (5).

Note that some algorithms are developed in the case $C \neq 0$ and some other algorithms are developed in the case $C=0$ using a normalization assumption such as the knowledge of the static gain or the knowledge that $\left\|\theta^{*}\right\|_{2}=1$ (see for instance [18], [3], [16], [7] and so on). This paper is presented in the case $C=0$, it must be noticed that all the algorithms presented here can be adapted to the case $C \neq 0$. 


\section{Identification algorithms}

\subsection{Method 1: Identification algorithm of [9]}

A first algorithm is proposed in [9] in 2006. This algorithm is based on the estimation of $\left\{x_{t}\right\}_{t=1}^{N}$ in a first time.

Main assumptions in [9] are the following:

- The noise $n_{t}$ is a sequence of independent identically distributed (i.i.d.) random variables. The inverse $F^{-1}($.) of its distribution function $F($.$) is assumed to be known$

- The input sequence $u_{t}$ is n-periodic.

The algorithm proposes the estimation of $\theta^{*}$ every $n$ samples. The proposed algorithm is a two steps algorithm:

- Step 1: estimation of the output of the system $G^{*}(q)$ based on $s_{t}$, i.e. estimation of $\left\{x_{t}\right\}_{t=1}^{N}$. The estimation is realized recursively every $n$ samples using the inverse of the distribution function of the noise. $\widehat{x}_{t}$ denotes the estimate of $x_{t}$.

- Step 2: estimation of $\theta^{*}$ using the input $u_{t}$ and the estimated output of the first step. In this second step, the estimate of $\theta^{*}$ is given by:

$$
\widehat{\theta}_{t}=\left(\widehat{\Phi}_{t} \widehat{\Phi}_{t}^{T}\right)^{-1} \widehat{\Phi}_{t} \widehat{X}_{t}
$$

where

$$
\begin{aligned}
& -\widehat{X}=\left(\begin{array}{c}
\widehat{x}_{t} \\
\vdots \\
\widehat{x}_{t+n a+n b}
\end{array}\right) \\
& -\widehat{\Phi}=\left(\begin{array}{lll}
\widehat{\phi}_{t} & \cdots & \widehat{\phi}_{t+n a+n b}
\end{array}\right) \text { with } \widehat{\phi}_{t}=\left(\begin{array}{c}
-\widehat{x}_{t-1} \\
\vdots \\
-\widehat{x}_{t-n a} \\
u_{t} \\
\vdots \\
u_{t-n b}
\end{array}\right)
\end{aligned}
$$

Note that a convergence analysis is provided in [9].

\subsection{Method 2: Identification algorithm of [10]}

The algorithm presented in [10] is a Quadratic Programming-based method. The estimate of $\theta^{*}$ is obtained by solving the QP problem defined by:

$$
\underset{\min _{M}}{\text { s. t. } M_{L} \leq M \leq M_{H}} M^{T} H M
$$

where

- $M=\left(\begin{array}{c}\theta \\ X\end{array}\right)$

- $M_{L}$ and $M_{H}$ are lower and upper bounds of $M$, these bounds are used to take into the binary sensor $Q_{C}($.$) .$ 
- $H=\left(\begin{array}{cc}\Phi \Phi^{T} & -\Phi \\ -\Phi^{T} & I_{N}\end{array}\right)$

- $X=\left(\begin{array}{c}x_{1} \\ \vdots \\ x_{N}\end{array}\right)$

$\Phi=\left(\begin{array}{lll}\phi_{1} & \cdots & \phi_{N}\end{array}\right)$ with $\phi_{t}=\left(\begin{array}{c}-x_{t-1} \\ \vdots \\ -x_{t-n a} \\ u_{t} \\ \vdots \\ u_{t-n b}\end{array}\right)$

From the fact that $x_{t}$ is unknown, an iterative algorithm is used in [10]. This algorithm is the following:

- Initialization step: $\phi_{t}$ is initialized as follows $\phi_{t}=\left(\begin{array}{c}0 \\ \vdots \\ 0 \\ u_{t} \\ \vdots \\ u_{t-n b}\end{array}\right)$. This initialization step provides a first estimate of $\theta^{*}$ and $X$

- Iteration step: in the $i^{\text {th }}$ iteration, $\phi_{t}$ is built using the estimate of $X$ obtained at the previous iteration.

\subsection{Method 3: Identification algorithm of [14]}

The algorithm presented in [14] is dedicated to identification of Wiener systems, where the relation between $y_{t}$ and $x_{t}$ is given by:

$$
y_{t}=g\left(x_{t}+n_{t}\right)
$$

with $g($.$) is a static function. In this paper we consider the case g(x)=x$.

The algorithm is based on the following assumptions:

- The input $u_{t}$ is symmetrical independent identically distributed (i.i.d).

- $n_{t}$ is a bounded white noise.

The algorithm proposed in [14] also is a two-step algorithm:

- Step 1: estimation of a high order FIR model, i.e. estimation of the impulse response. Denote $\theta^{i m p} \in R^{m}$ the parameters of the impulse response and $\phi_{t}^{i m p} \in R^{m}$ the associated observation vector. The Support Vector Machine algorithm is used to estimate the parameter vector of the hyperplan which separates observation vectors providing an output superior to $C$ from observation vectors providing an output inferior to $C$. The estimate of $\theta^{i m p}$ is obtained by solving the following problem

$$
\underset{\text { s. t. } s_{t}\left(\left(\phi_{t}^{i m p}\right)^{T} \theta^{i m p}\right) \geq 1}{\min _{\theta^{i m p}}} \frac{1}{2}\left\|\theta^{i m p}\right\|^{2}
$$


- Step 2: estimation of $\theta^{*}$ using the estimated impulse response. This step correspond to a model reduction step.

Note that a convergence analysis is provided in [14].

\subsection{Method 4: Identification algorithm of [12] and [13]}

In [12] and [13] a recursive identification algorithm is proposed using the maximum likelihood criterion.

The algorithm presented in this article is based on the following main assumptions:

- $n_{t}$ is i.i.d sequence with normal distribution: its distribution function is known.

The algorithm presented in [12] and [13] is recursive algorithm with two successive steps. Technical details for each step are provided in [12] and [13]. These two steps are the following:

- The first step is an initialization step. The parameter vector $\theta^{*}$ is estimated using an online algorithm based on the expectation maximization (EM) method. The EM method has a slow convergence rate, this first step is used for few iterations.

- The second step an online algorithm based on the quasi-Newton method. The estimate with the EM method is used for the initialization.

Note that a convergence analysis is provided in [13].

\subsection{Method 5: Identification algorithm of [11]}

The identification algorithm presented in [11] is based on the Stochastic Approximation Algorithms With Expanding Truncations (SAAWET). The algorithm of [11] is based on the following assumptions:

- The input sequence $u_{t}$ is an i.i.d Gaussian sequence.

- The additive noise $n_{t}$ is zero mean Gaussian noise.

The algorithm of [11] is a two-step algorithm organized as follows:

- Step 1: as in [14] the system is approximated by a high order FIR model. The parameter of the impulse response, i.e. the parameter of the high order FIR model, are estimated recursively using the SAAWET.

- Step 2: estimation of $\theta^{*}$ using the estimated impulse response. This step correspond to a model reduction step.

Note that a convergence analysis is provided in [11].

\subsection{Method 6: Identification algorithm of [15]}

The last algorithm in this study, is the one presented in [15]. The algorithm is based on use the Filtered Output Error Optimal Bounding Ellipsoid (F-OE-OBE) algorithm presented. In the case $C=0$, the objective of the algorithm proposed in [15] is to realize the estimation of a model such that its output has the same sign than $s_{t}$. The main assumption of the algorithm of [15] is the following:

- The noise $n_{t}$ is with zero mean, it is wide sense stationary and uncorrelated with $u_{t}$.

The algorithm presented in [15] is an iterative algorithm. This algorithm is the following: 


\begin{tabular}{|c|c|c|c|c|c|c|}
\hline method & 1 & 2 & 3 & 4 & 5 & 6 \\
\hline \hline failure rate & $1 \%$ & $0 \%$ & $0 \%$ & $26 \%$ & $0 \%$ & $0 \%$ \\
\hline
\end{tabular}

Table 1. Failure rate for each method.

- Initialization step: initialization with the OE-OBE algorithm or using the least square algorithm (from the knowledge of $s_{t}$ ).

- Iteration step: in the $i^{\text {th }}$ iteration, use of the F-OE-OBE algorithm, the filter is built using the model estimated at the previous iteration.

Note that a convergence analysis is provided in [15].

\subsection{Particular algorithm}

There are an other algorithm proposed in literature for the identification of IIR system using quantized or binary output. The algorithm presented in [17] studies the identification of ARX model. The noise is assumed to be bounded.

\section{Comparison and numerical simulation}

Tab.2 summarizes some characteristics of the previous algorithm: the main assumptions on the input signal,the output signal, and the noise, type of algorithm and if there is a convergence analysis.

Some simulation results are reported in this section so as to compare some aspects of the previous algorithms. It is difficult to provide general conclusions from a numerical example, however we can observe some interesting first behaviors.

Numerical data have been generated according to (1) and (5) with $G^{*}(q)$ given by:

$$
G^{*}(q)=\frac{1-1.4599 q^{-1}-0.7299 q^{-2}}{1-1.1254 q^{-1}+0.5383 q^{-2}}
$$

The threshold is chosen equal to $C=0$.

In a first experiment we test the six previous algorithms in a reference framework. The input sequence $u_{t}$ is a zero mean random sequence with normal distribution and an unitary standard deviation. The number of available data is $N=1000$. The noise $n_{t}$ is a zero mean random sequence with normal distribution such that signal to noise (SNR) is SNR $=20 d B$.

User parameters of each algorithm are chosen in order to have the minimal error $E=$ $\left\|\theta^{*}-\operatorname{mean}(\widehat{\theta})\right\|$ on a Monte Carlo simulation carried out with 100 runs. The obtained user parameters are the following:

- for [9]: we use the periodic sequence $\{-0.5 ; 0.8 ;-0.1 ; 0.4 ; 0.9\}$.

- for [10]: we use the "quadprog" function of MATLAB over 20 iterations.

- for [14]: we estimate the 30 first parameters of the impulse response.

- for [13]: we apply the quasi-Newton method after $50 \%$ of samples.

- for [11]: we estimate the 30 first parameters of the impulse response and $M_{t}=6^{t}$ (the user parameter of the algorithm); 

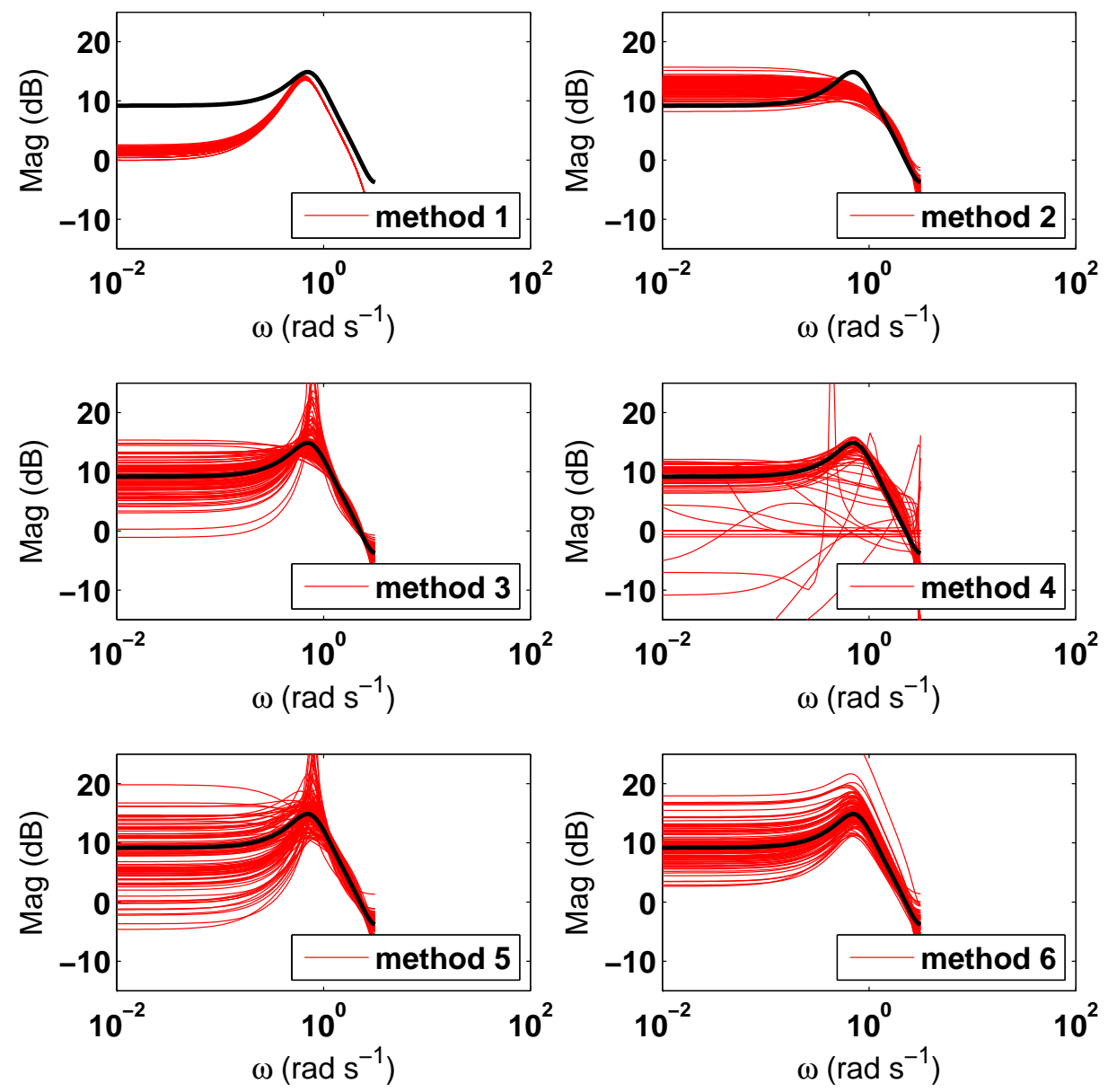

Figure 2. Comparison of Bode plot for each algorithm.

- for [15]: we apply the F-OE-OBE over 20 iterations with $\lambda=0.99, \delta=0.99$ (the user parameters of the algorithm).

The bode plot of each estimated model is given on Fig. 2 in red, the real system is in black. Note that it is possible on some data sets that an algorithm fails to provide a solution or provide an unstable solution. Tab. 1 presents the failure rate for each method. In the present context, it appears from these results that:

- method 1 and 2 provide biased estimates.

- method 4 provides on some data sets good estimates but the failure rate is too important.

- method 3, 5 and 6 have best performances. The lower variance is obtained with method 6. 


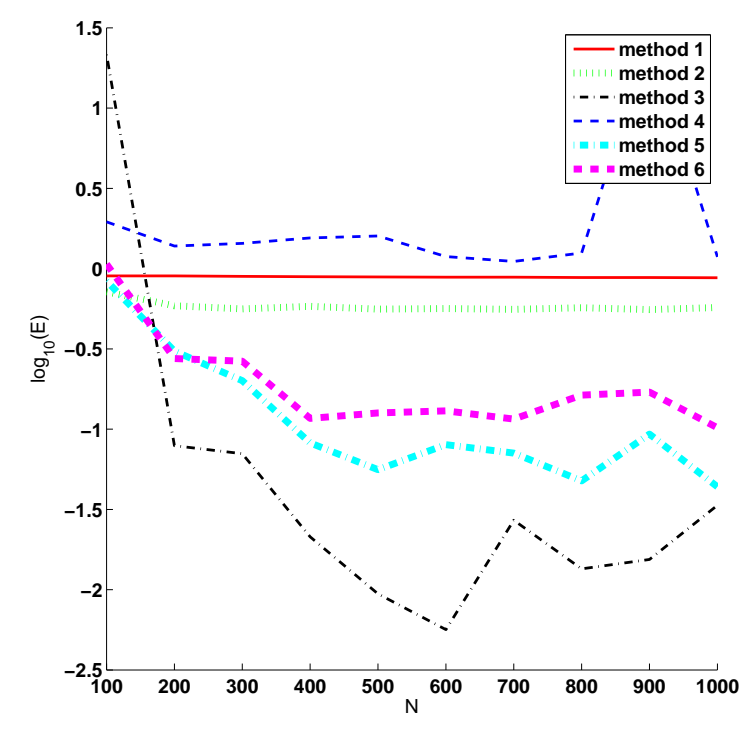

Figure 3. Impact of the number of available data on performance.

In a second experiment we test the impact of the number of available data. Fig. 3 presents $E=\left\|\theta^{*}-\operatorname{mean}(\widehat{\theta})\right\|$ for each algorithms as a function of $N$ for $N$ from 500 to 5000 . These results are obtained with Monte Carlo simulations carried out with 100 runs. It appears that:

- performances of method 1 , method 2 , and method 4 are not very sensitive to a high number of data.

- performances of method 3, method 5 and method 6 depend on the number of available data. For a high number of data, these methods allow a better identification of the system.

In a third experiment we test the impact of the noise on performance of the six algorithms. Several SNR have been tested: from $0 d B$ to $40 d B$. Fig. 4 presents $E$ for each algorithm as a function of SNR. Here again these results are obtained with Monte Carlo simulations carried out with 100 runs. It appears that:

- performances of method 1 are better for low SNR.

- performances of method 3, method 5 and method 6 depends on the SNR data. For a high SNR these methods allows

\section{CONCLUSION}

In this paper, an attempt has been made To compare the wide range of methods dedicate to identification of IIR systems using only binary or quantized output, that has appeared over the last decade. Based on experimental data, several characteristics of the six methods have been tested, as sensitivity to noise, impact of the number of available data.

This short description, and comparison must be extended. More detail in presentation of the algorithms, and the comparison in other context can be subject of future works. 


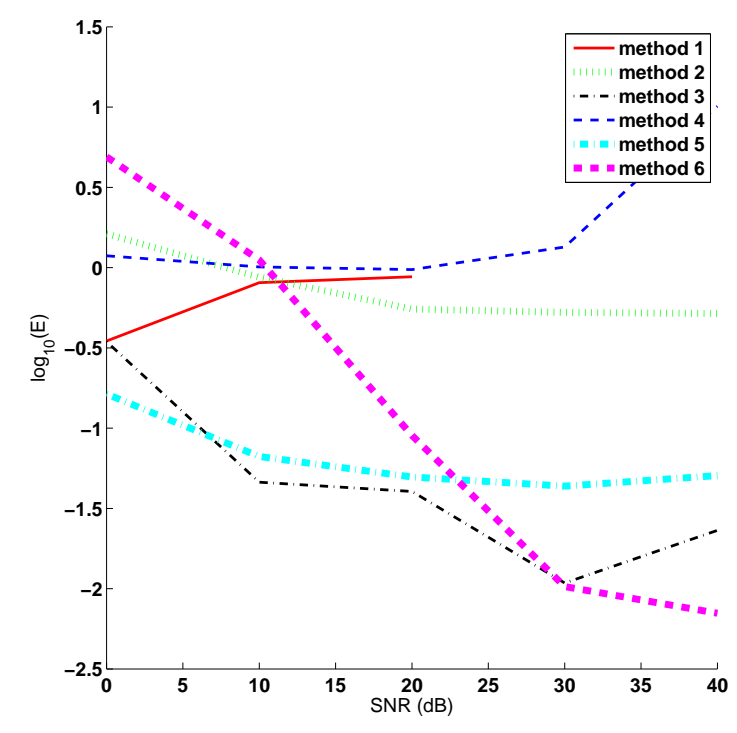

Figure 4. Impact of the noise on performance.

\section{References}

[1] Y. Zhao, L.Y. Wangb, G. GeorgeYin \& J.F. Zhang. "Identification ofWiener systems with binary-valued output observations", Automatica 43, 1752-176, (2007).

[2] E. Colinet, J. Juillard: "A Weighted Least-Squares Approach to Parameter Estimation Problems Based on Binary Measurements", "IEEE Transactions on Automatic Control 55, 1, 148-152 (2010).

[3] K. Jafaridinani, J. Juillard \& M. Roger. "Convergence Analysis of an online Approach to Parameter Estimation Problems Based on Binary Observations", Automatica, International Federation of Automatic Control, 48 (11), pp.2837-2842, (2012).

[4] J. Guo and Y. Zhao: "Recursive projection algorithm on FIR system identification with binary-valued observations", Automatica 49, 3396-3401, (2013).

[5] J. Guo, Y. Zhao, C.Y. Sun \& Y. Yu: "Recursive identification of FIR systems with binaryvalued outputs and communication channels", Automatica 60, 165-172, (2015).

[6] A. Goudjil, M. Pouliquen, E. Pigeon, O. Gehan \& M. M'Saad: "Identification of systems using binary sensors via support vector machines",IEEE Conference on Decision and Control, Osaka, (2015).

[7] M. Pouliquen, T. Menard, E. Pigeon, O. Gehan and A. Goudjil. "Recursive System Identification Algorithm using Binary Measurements",European Control Conference, Aalborg, (2016).

[8] J. Guo, L. Y. Wang, G. Yin, Y. Zhao \& Ji-Feng Zhang: "Identification of Wiener systems with quantized inputs and binary-valued output observations", Automatica 78, 280-286, (2017).

[9] L.Y. Wanga, G.G.Yin and J.F. Zhang. "Joint identification of plant rational models and noise distribution functions using binary-valued observations", Automatica 42, 535-547, 
(2006).

[10] X. Liu, J. Wang and Q. Zhang. "A quadratic programming-based method for quantized system identification", Proceedings of the 18th World Congress The International Federation of Automatic Control Milano (Italy) August 28 - September 2, (2011).

[11] Qijiang Song. "Recursive identification of systems with binary-valued outputs and with ARMA noises", Automatica 93, 106-113, (2018).

[12] D. Marelli, K. You and M. Fu. " Identification of ARMA models using intermittent and quantized output observations", International Conference on Acoustics, Speech and Signal Processing, (2011).

[13] D. Marelli, K. You, M. Fu. "Identification of ARMA models using intermittent and quantized output observations", Automatica 49, 360-369, (2013).

[14] G. Li and C. Wen. "Identification of Wiener Systems With Clipped Observations", IEEE Transactions on Signal Processing, VOL. 60, NO. 7, JULY (2012).

[15] M. Pouliquen and E. Pigeon and O. Gehan and A. Goudjil. "Identification using Binary Measurements for IIR Systems",IEEE Transactions on Automatic Control, 55,148-152 (2019).

[16] L. Bourgois, J. Juillard. "Convergence Analysis of an Online Approach to Parameter Estimation Problems Based on Binary Noisy Observations". CDC'12, Maui, Hawai, United States. pp.1506-1511, (2012).

[17] M. Casini, A. Garulli \& A. Vicino: "Set-membership identification of ARX models with quantized measurements", 2011 50th IEEE Conference on Decision and Control and European Control Conference (CDC-ECC) Orlando, FL, USA, December 12-15, (2011).

[18] K. Jafari, J. Juillard and E. Colinet: "A Recursive System Identification Method Based on Binary Measurements", 49th IEEE Conference on Decision and Control December 15-17, 2010, Hilton Atlanta Hotel, Atlanta, GA, USA, (2010).

[19] L.Y. Wang, J.F. Zhang and G. Yin. "System Identification Using Binary Sensors", IEEE Transactions on Automatic Control, VOL. 48, NO. 11, NOVEMBER (2003). 


\begin{tabular}{|c|c|c|c|c|c|}
\hline Algorithm reference's & $\begin{array}{l}\text { Algorithm } \\
\text { type }\end{array}$ & $\begin{array}{l}\text { The input } \\
u_{t}\end{array}$ & $\begin{array}{l}\text { The out- } \\
\text { put } s_{t}\end{array}$ & The noise $n_{t}$ & $\begin{array}{l}\text { Convergence } \\
\text { analysis }\end{array}$ \\
\hline [9] & recursive & $\begin{array}{l}n- \\
\text { periodic }\end{array}$ & Binary & $\begin{array}{l}\text { i.i.d, } \quad F(.) \\
\text { and } f(.) \text { are } \\
\text { known. }\end{array}$ & Yes. \\
\hline [10] & iterative & 一 & Quantized & 一 & No. \\
\hline [14] & batch & i.i.d & Binary & $\begin{array}{l}\text { Bounded } \\
\text { white noise }\end{array}$ & Yes. \\
\hline$[12] \&[13]$ & recursive & - & Quantized & $\begin{array}{l}\text { i.i.d }, F(.) \\
\text { and } f(.) \text { are } \\
\text { known. }\end{array}$ & Yes. \\
\hline [11] & recursive & $\begin{array}{l}\text { i.i.d } \\
\text { Gaussian }\end{array}$ & Binary & $\begin{array}{l}\text { Zero mean } \\
\text { Gausian }\end{array}$ & Yes. \\
\hline [15] & iterative & - & Binary & $\begin{array}{l}\text { Zero mean } \\
\text { with wide } \\
\text { sense } \\
\text { stationary } \\
\text { and uncor- } \\
\text { related with } \\
\text { the input }\end{array}$ & Yes. \\
\hline
\end{tabular}

Table 2. Summary of some characteristics 